\section{Die SANZ verabschiedet sich definitiv per Ende Mai 2001: Eine unglaubliche Geschichte}

M. Giger

Unerwünschte Arzneimittelwirkungen wie diejenigen von Thalidomid, Eraldin und Mexaform mit schwersten Folgen führten Ende 1979 zur Gründung der Stiftung Schweizerische Arzneimittelnebenwirkungszentrale SANZ. Zwar wäre die Pharmakovigilanz auch in den Aufgabenbereich der Registrierungsbehörde (IKS) gefallen; diese hatte zu jener Zeit wenig Gehör und Interesse für diesen wichtigen Zweig der Arzneimittelsicherheit. Mit dem Ziel, Meldungen von Ärztinnen und Ärzten über vermutete unerwünschte Arzneimittelwirkungen zu sammeln und auszuwerten, betrauten die Stifter, die Verbindung der Schweizer Ärztinnen und Ärzte FMH und die Schweizerische Gesellschaft für Chemische Industrie SGCI, Prof. Dr. med. Johannes Gartmann mit dem Aufbau einer Sammelstelle in Chur. Ihm stand ein wissenschaftlicher Beirat zur Seite. Im Oktober 1990 übernahm Dr. med. Max Kuhn zusammen mit dipl. pharm. Katharina Hartmann die Leitung der Churer Sammelstelle. Die eingehenden Meldungen wurden innerhalb dreier Arbeitstage mit einer Beurteilung den meldenden Medizinalpersonen und den Vertriebsfirmen zugestellt. Die Vertriebsfirmen steuerten daraufhin die firmeninternen Daten bezüglich Arzneimittel, die mit den unerwünschten Wirkungen in Zusammenhang standen, zu. So erfolgte in Kenntnis aller verfügbaren Daten einige Wochen später die endgültige Beurteilung der Kausalität. Rasch erfreute sich die SANZ dank ihrer speditiven und kompetenten Arbeit grosser Beliebtheit in Ärzte- und Apothekerschaft. Dank ihrer Fachkompetenz stiess die SANZ in steigendem Ausmass bei den Behörden und in wissenschaftlichen Kreisen auf Anerkennung. Die SANZ wurde zu einem weit herum geschätzten Referenzzentrum für Pharmakovigilanz.

Korrespondenz:

Dr. med. Max Giger

Stiftungsrat SANZ

Oberer Deutweg 59

CH-8400 Winterthur
Erfolg hat nicht nur Bewunderer. Schon bald wurde von verschiedener Seite der Vorwurf der Industrieabhängigkeit laut und ist bis heute nicht verstummt. Damals wie heute zu Unrecht: Von Anfang an führte der ärztliche Leiter die SANZ selbständig im Auftrag des Stiftungsrates, in welchem auch nach Erweiterung der Trägerschaft um die Firmen des Verbandes der Importeure Pharmazeutischer Spezialitäten VIPS sowie den Schweizerischen Apothekerverband SAV die Verbandsvertreter der blauen Berufe immer die Stimmenmehrheit hatten. Um diesen Vorwurf aber auch in der Öffentlichkeit demonstrativ zu entkräften, beschloss der Stiftungsrat 1991, die SANZ weiter $\mathrm{zu}$ öffnen: die Kommunikation von unerwünschten Arzneimittelwirkungen aus der SANZDatenbank lag neu einzig in der Kompetenz des ärztlichen Leiters, des weiteren wurde die Datenbank Drittpersonen, v.a. Dissertantinnen und Dissertanten zur wissenschaftlichen Auswertung zugänglich gemacht. Es folgten als Ausdruck der offenen Kommunikation die SANZ-Streiflichter, in welchen über aktuelle praxisrelevante Nebenwirkungsprobleme berichtet wurde.

Im zehnten Jahr erst ihres Bestehens, im August 1991, konnte die SANZ mit der IKS eine Zusammenarbeitsvereinbarung schliessen, die im Januar 1995 verlängert wurde. Aus dieser Vereinbarung entstanden der IKS keine finanziellen Verpflichtungen; es wurde einzig der Datenaustausch und eine Zusammenarbeit auf wissenschaftlicher Basis im Interesse einer einheitlichen schweizerischen Pharmakovigilanz vereinbart. Dieser Pharmakovigilanz-Rat bzw. das Pharmakovigilanz-Board kam leider nie zum Tragen. Ende 1995 vereinbarte die SANZ mit dem damaligen Blutspendedienst des Schweizerischen Roten Kreuzes den Aufbau einer Hämovigilanz, um unerwünschte Reaktionen auf labile Blutprodukte zu erfassen und dem Blutspendedienst so Hinweise zur Qualitätssicherung zu vermitteln. Die Abteilung für Impffragen des Bundesamtes für Gesundheit BAG beauftragte im Sommer 1999 die SANZ mit der Übermittlung der Meldungen von Impfreaktionen; dies aus der Tatsache, dass bei der SANZ mehr als doppelt so viele Meldungen eingingen als bei den Kantonsärztlichen Diensten als obligatorische Meldestellen.

Trotz dieser zunehmenden Beliebtheit und wissenschaftlich anerkannten Stellung der SANZ wurden schon in den frühen Diskussionen um den Entwurf zum Heilmittelgesetz, d.h. in den ersten Monaten des Jahres 1995, Stimmen laut, die ein weiteres Überleben der SANZ in Frage stellten und eine andere, zentralistische staatliche Pharmakovigilanz forderten. Durch das von den Räten im letzten Jahr verabschiedete Heilmittelgesetz soll im Rahmen des Heilmittelinstitutes der Pharmakovigilanz anerkanntermassen eine wichtigere Stellung beigemessen werden. Das Melden von vermuteten unerwünschten Arzneimittelwirkungen soll obligatorisch werden. 
Vergehen gegen das Gesetz werden dem Strafgesetz unterstellt. Wie so üblich bei zentropetalen Tendenzen soll auch die sogenannte Fachkompetenz ins Heilmittelinstitut verlagert werden. Die SANZ sollte zusammen mit den universitären Instituten für Klinische Pharmakologie und Toxikologie einzig noch als periphere Sammelstation, quasi als Briefkasten für die meldenden Medizinalpersonen, dienen. Serviceleistungen, nebst dem Vertrauen der meldepflichtigen Medizinalpersonen in die Betreiber der Meldestelle ein unabdingbares Stimulans für die Meldefreudigkeit, wurden (und werden) als überflüssig erachtet. Zudem sollte die SANZ für diese Briefkastenfunktion mit 150000 Franken pro Jahr auskommen, wohlgemerkt bei gleichzeitigem Verzicht auf die Beiträge der Industrie (zwecks Wahrung der Unabhängigkeit), was einer Reduktion des Jahresbudgets um rund 70\% entspricht. Die Mehrkosten wären nach Ansicht der Behörden einfach durch die Berufsverbände zu tragen. Im Klartext: Die Meldepflichtigen sollen ihre gesetzliche Pflicht gleich auch noch selber finanzieren. Ein eigenwilliges Staatsverständnis, das die Strategen in der Bundesverwaltung hier an den Tag legen. Die FMH hat diesem Staatsverständnis eine Absage erteilt. Nachdem die FMH Jahr für Jahr gut 20\% an das Budget der SANZ beigetragen hat, beschloss die Ärztekammer im Jahr 2000, die SANZ nur noch bis zum Inkrafttreten des Heilmittelgesetzes finanziell zu unterstützen.
Aufgrund dieser Entwicklung musste der Stiftungsrat die Schliessung der Churer Sammelstelle auf Ende Juni 2001 in die Wege leiten und der Aufsichtsbehörde die Aufhebung der Stiftung beantragen. Im Rahmen dieses Aufhebungsverfahrens «klagten" dieselben Behördenvertreter, welche die SANZ mit 150000 Franken abspeisen wollten, dass wegen des Verschwindens dieser anerkannten Institution der Personaletat im künftigen Heilmittelinstitut um drei Personen (sic!) aufgestockt werden müsse. Dies dürfte effektiven jährlichen Kosten von rund 450000 Franken entsprechen, also recht genau dem Jahresbudget der SANZ! Honi soit qui mal y pense ...

Was bleibt, ist die Erinnerung an eine zwanzigjährige bestens funktionierende privatwirtschaftlich betriebene Sammelstelle in Chur: Über 18000 Meldungen wurden erfasst und verarbeitet. Die bezüglich Arzneimittelsicherheit relevanten Meldungen wurden an die Registrierungsbehörde weitergeleitet. Viele dieser Meldungen veranlassten die Behörden zur Einleitung von Massnahmen zur Steigerung der Arzneimittelsicherheit. Dem eingespielten und aufgestellten Churer Team gilt der aufrichtige und herzliche Dank für den jahrelangen unermüdlichen Einsatz im Interesse der Arzneimittelsicherheit! 